\title{
Spatial-time Visualization of Cutaneous Leishmaniasis in an Urban Area: Informing Health Policy and Practice
}

\section{Neda Firouraghi}

Mashhad University of Medical Sciences

\section{Sayyed Mostafa Mostafavi}

Mashhad University of Medical Sciences

Amene Raouf-Rahmati

Mashhad University of Medical Sciences

Alireza Mohammadi

University of Mohaghegh Ardabili

\section{Reza Saemi}

Mashhad University of Medical Sciences

Mahmoud Fakher

Mashhad University of Medical Sciences

\section{Davidson H Hamer}

Boston University School of Medicine

\section{Elham Moghaddas}

Mashhad University of Medical Sciences

Behzad Kiani ( $\sim$ Kiani.Behzad@gmail.com )

Mashhad University of Medical Sciences

\section{Research}

Keywords: Cutaneous leishmaniasis, spatial empirical Bayesian, cluster analysis, geographical information systems, spatial-time analysis

Posted Date: December 12th, 2020

DOl: https://doi.org/10.21203/rs.3.rs-125091/v1

License: (c) (1) This work is licensed under a Creative Commons Attribution 4.0 International License. Read Full License 


\section{Abstract}

Background:

Cutaneous leishmaniasis $(\mathrm{CL})$ is an important public health concern worldwide. Iran is among the most CL-affected countries, being listed as one of the first six endemic countries in the world. In order to develop targeted interventions, we performed a spatial-time visualization of CL cases in an urban area to identify high-risk and low-risk areas during 2016-2019.

Methods:

This cross-sectional study was conducted in the city of Mashhad. Patient data were gathered from Mashhad health centers. All cases $(n=2425)$ were diagnosed in two stages; the initial diagnosis was based on clinical findings. Subsequently, clinical manifestation was confirmed by parasitological tests. The data were aggregated at the neighborhood and district levels and smoothed CL incidence rates per 100,000 individuals were calculated using the spatial empirical Bayesian approach. Furthermore, we used the Anselin Local Moran's / statistic to identify clusters and outliers of CL distribution during 2016-2019 in Mashhad.

Results:

The overall incidence rates decreased from 34.6 per 100,000 in 2016 to 19.9 per 100,000 individuals in 2019. Both cluster analyses by crude incidence rate and smoothed incidence rate identified high-risk areas in southwestern Mashhad over the study period. Furthermore, the analyses revealed low-risk areas in northeastern Mashhad over the same 3-year period.

Conclusions:

The southwestern area of Mashhad had the highest CL incidence rates. This piece of information might be of value to design tailored interventions such as running effective resource allocation models, informed control plans and implementation of efficient surveillance systems. Furthermore, this study generates new hypotheses to test potential relationships between socio-economic and environmental risk factors and incidence of $\mathrm{CL}$ in areas with higher associated risks.

\section{Background}

Leishmaniasis, a serious worldwide public health problem caused by intracellular protozoan parasites, was prevalent in 92 countries in 2018 with an estimated 700,000 to 1 million new annual infections $(1,2)$. It is a vector-borne disease with a wide range of symptoms, which is transmitted to humans by phlebotomine sand flies (3). There are different variants of this disease, the most common of which is the cutaneous form. Over $85 \%$ of new identified cutaneous leishmaniasis (CL) cases originated in Afghanistan, Algeria, Brazil, Colombia, Iran (Islamic Republic of), Pakistan, Peru, and the Syrian Arab Republic in $2018(2,4)$. Recently, the number of CL cases has significantly increased in the endemic 
countries (5). This increase is attributed to changes in both natural and human-made environments such as fast and unplanned urbanization, increase in agricultural development, large migrations, and deforestation $(6,7)$. CL causes psychological, social, and economic problems (8), and is a major public health challenge in the affected areas.

Iran is among the top six countries in the world with the highest annual rate of CL incidence $(2,4)$, with 50-250 cases per 100,000 individuals (9). CL, the second most prevalent arthropod-transmitted disease (10), is a complex public health problem and its distribution follows a spatial pattern in Iran $(2,4,11)$. The Khorasan-Razavi province, located in northeastern Iran, has the highest prevalence of $C L$ in the country $(12,13)$. Mashhad, the capital of Khorasan-Razavi province, is the second most populous city in Iran and is bordered by two neighboring CL-prevalant countries, Turkmenistan and Afghanistan. Moreover, Mashhad, one of the main CL endemic cities $(14,15)$, has different $C L$ incidence rates across different geographical areas (16).

Identification of geographical distribution and spatio-temporal patterns of disease can help to implement effective preventive strategies. Geographic information system (GIS) has been previously applied to visualize the geographical distributions of disease and the spatial modelling of risk factors, and in particular environmental factors $(17,18)$. GIS is a powerful tool to gather and analyze spatial and nonspatial data regarding the epidemiology of disease and understanding its underlying ecology. Spatial and spatial-time analyses can provide insight into disease occurrence and guide potential tailored disease control interventions. As such, identifying high-risk areas can be useful to implement surveillance programs and come up with effective disease control strategies $(19,20)$. Because environmental factors can potentially impact the geographical distribution of leishmaniasis, using spatial analysis can foster valuable insight for health policymakers (21).

According to previous studies, GIS-based techniques led to the detection of countries with the highest rate of CL cases between 2001 and 2011 in Latin America (22). In a study conducted in Brazil, the areas with high $C L$ incidence rate were identified using spatial analysis (23). A number of studies used spatial analysis to assess spatial distribution and pattern of CL in some areas of Iran at provincial level (24-28). Furthermore, other studies have attempted to determine relations between epidemiological and environmental factors and $C L$ incidence in endemic areas $(24,27,29)$. A few studies have been carried out in Khorasan provinces (North, Razavi and South) to assess the spatial distribution of CL at provincial and national levels. In these studies, southern areas of North Khorasan province and northern areas of Razavi Khorasan province were identified as hot areas $(28,30)$. Another study revealed the high prevalence of $C L$ in southwestern areas of Mashhad using molecular methods (14). An epidemiological study assessed CL prevalence in Mashhad over a period of 20 years (1992-2014) and identified climaterelated factors with remarkable influence on the prevalence of $\mathrm{CL}$ (31). The previous studies confirmed the high prevalence of $\mathrm{CL}$ in Mashhad, but to the best of our knowledge, no study has investigated the spatial-time pattern of $\mathrm{CL}$ incidence at district and neighborhood levels in Mashhad. This underscores the need to explore the geographical distribution and high-risk disease areas. Exploring high-risk areas is an important step in developing strategies to effectively allocate healthcare resources, so as to overcome the 
disease and its associated complications (32). The objective of the present study is to apply a spatial analysis of CL cases in the city of Mashhad between 2016 and 2019, and to identify the spatial-time pattern of CL cases and identify the critically affected areas.

\section{Methods}

\section{Study area}

This cross-sectional study was conducted in the city of Mashhad, located in northeastern Iran (Fig. 1) with an approximate population of 3,000,000. Mashhad is situated at a latitude of $35^{\prime} \mathrm{N}-37^{\prime} \mathrm{N}$ and longitude of $59^{\prime} \mathrm{E}-60^{\prime} \mathrm{E}$, with a total area of $328 \mathrm{~km}^{2}$.

\section{Cutaneous leishmaniasis definition and diagnosis methods}

Cutaneous leishmaniasis is the most common form of leishmaniasis, which causes skin ulcers and serious disabilities persisting for months and sometimes for years. The skin lesions develop in the exposed parts of the body $(2,33)$. CL is caused by parasites, transmitted by the bite of phlebotomine sandflies, and affects humans (3). Diagnosis of $C L$ is through a combination of clinical symptoms and laboratory validation (2). In this study, all cases were diagnosed in two stages. This includes an initial diagnosis based on clinical examination followed by a validation using parasitological tests.

\section{Data sources}

Data were obtained from different sources at different spatial scales. Individual CL data were obtained from five Mashhad health Centers, including patient data collected between 2016 and 2019. In the past years, CL data were generally gathered at the municipal level. However, during the study period, data collection was performed at district and neighborhood scales. The base maps for conducting spatial analyses were obtained from Mashhad municipality.

\section{Data preparation}

All patients' addresses were geocoded manually using Google's MyMaps software (https://www.

google.com/maps/about/mymaps). In order to generate the point map (Fig. 1) and protect patients' privacy, we used a geomasking technique. Each patient's address was randomly assigned to a location within a radius of half a kilometer around their actual home address.

\section{Descriptive mapping}

To generate descriptive maps, we used the natural break classification with five classes. This classification method produces the best arrangement of values into different classes by minimizing the class's average deviation from the class mean. This method aims to reduce the variance within classes and increase the variance between classes (34).

\section{Empirical Bayesian Smoothing}


The relevant population at risk typically varies across the areas, so the precision of raw incidence rate will vary as well. This variance instability requires the smoothing of rates. The main objective in CL mapping was to estimate the incidence rate for each geographical unit using the observed number of patients. Empirical Bayes is one of the spatial statistical smoothing methods which is used to reduce the random fluctuations due to population size. In this model, the estimation of underlying rates is attained by making an assumption about prior disease distribution using observed data (35-37). The empirical Bayes technique computes the risk as a weighted sum of the raw rate for each unit and a prior mean (38, 39). The incidence rate of CL during 2016-2019 was adapted for smoothing by the Bayesian models.

\section{Spatial cluster analysis}

Mashhad has 172 neighborhoods and 13 districts. The population of all neighborhoods and districts was obtained from the Statistics Center of Iran. The Local Moran's / statistic (40) was performed to identify potential spatial-time clusters in terms of CL incidence rate during the 2016-2019 period at the neighborhood level. We used two incidence rates for spatial clustering: raw incidence rate and empirical Bayesian incidence rate. We computed the leishmaniasis raw incidence rate for each geographical unit by dividing leishmaniasis cases in each unit by its total population at risk in that unit. The use of local Moran's / clustering method has been suggested in many studies $(24,41,42)$. This model calculates a zscore and p-value to determine whether the apparent similarity (a spatial clustering of either high or low values) or dissimilarity (a spatial outlier) is more pronounced than the one expected in a random distribution. The null hypothesis states that CLs are randomly distributed across the study area. In this method, the areas with similar high or low CL rates are indicated by High-High (HH) and Low-Low (LL) areas, while the regions with dissimilar rates are described as High-Low (HL) and Low-High (LH) regions (43). In other words, the $\mathrm{HH}$ and $\mathrm{LL}$ areas indicate the presence of $\mathrm{CL}$ clusters, while $\mathrm{HL}$ and $\mathrm{LH}$ areas indicate outliers.

\section{Moran Scatter plot}

The Moran scatter plot consists of a plot with the original variable and spatially lagged variable. In this plot the spatial autocorrelation is classified into four categories that is visualized as four quadrants. Positive spatial autocorrelation (similar values in nearby or neighboring areas), known as $\mathrm{HH}$ and LL areas, are shown in the upper-right quadrant and the lower-left quadrants, respectively. In contrast, the lower-right and upper-left quadrants are related to negative spatial autocorrelation (unlike neighboring areas), known as $\mathrm{HL}$ and LH, respectively. The slope of scatter plot's line corresponds to Moran's / as the most common indicator of spatial autocorrelation. In this plot, the original variable has been standardized (zero mean and unit standard deviation). Also, the spatial lag variable is considered for those standardized values (44). In this study, the original variable was CL incidence and the spatially lagged variable was lagged incidence.

\section{Software}

We used Geoda (ASU, GeoDa Center for Geospatial Analysis and Computation, Arizona, USA) and ArcGIS, v. 10.6 (ESRI, Redlands, CA, USA) for spatial analyses and Microsoft Excel 2019 for the descriptive 
analyses.

\section{Results}

There were 2425 patient records collected during 2016-2019. These include 1047 cases in 2016-2017, 775 cases in 2017-2018 and 603 patients in 2018-2019. Table 1 shows the seasonal frequency of new $\mathrm{CL}$ cases. The frequency of CL cases decreased by 1047 to 603 from 2016 to 2019 . The CL incidence rates were $34.6,25.6,19.9$ per 100,000 during the three years, respectively. As the address was not registered for some patients and the population of some neighborhoods was not available, 282 cases were excluded from the spatial analysis. Finally, 2143 cases were included during 2016-2019.

Table 1

The number of new CL cases by season during 2016-2019 in the city of Mashhad, Iran

\begin{tabular}{|lcccccc|}
\hline & Spring & Summer & Autumn & Winter & $\begin{array}{l}\text { Total } \\
\text { Frequency }\end{array}$ & $\begin{array}{l}\text { CL Incidence (per } \\
\text { 10000) }\end{array}$ \\
\hline $\begin{array}{l}\text { March 2016-March } \\
2017\end{array}$ & 315 & 220 & 247 & 265 & 1047 & 34.6 \\
\hline $\begin{array}{l}\text { March 2017-March } \\
2018\end{array}$ & 239 & 177 & 184 & 175 & 775 & 25.6 \\
\hline $\begin{array}{l}\text { March 2018-March } \\
2019\end{array}$ & 177 & 135 & 162 & 129 & 603 & 19.9 \\
\hline
\end{tabular}

The temporal fluctuation of CL-positive cases is shown in Fig. 2. The total number of CL cases progressively decreased over the three-year period. The number of confirmed CL cases was highest in April-May during 2016-2019, while the lowest frequency was in August-September and February-March during 2016-2018 and 2018-2019, respectively. The results show seasonal variations in CL cases with high frequency in the spring. Disease frequency decreased during the summer, but the number of patients increased rapidly with the onset of autumn.

The highest incidence rates of CL cases at district levels were concentrated in the northwestern and southwestern areas of the city from 2016 to 2019. The reduction of CL incidence during the three years is clearly seen in the aforementioned areas (Fig. 3).

The descriptive maps of $\mathrm{CL}$ crude incidence at the neighborhood level revealed that the southwestern area of the city had the highest incidence rate. The local Moran's / revealed a statistically significant spatial autocorrelation regarding $\mathrm{CL}$ incidence at the neighborhood level. The cluster maps revealed $\mathrm{HH}$ clusters of CL in the southwestern area from March 2016 to March 2019. Furthermore, there was an LL cluster in the northeastern area of the city from March 2016 to March 2019 (Fig. 4). All clusters and outliers were statistically significant $(p<0.05)$.

As outlined in the Methods section, rates have an intrinsic variance instability, which may lead to the identification of spurious outliers. In order to correct for this, we can use smoothing approaches (also called shrinkage estimators), which improve the precision of crude rate by borrowing strength from other 
observations. The descriptive maps of smoothed incidence CL cases revealed that the southwestern area of the city was the area most affected by $\mathrm{CL}$ incidence. Also, the cluster maps confirm these results and show the HH clusters of CL cases in the southwestern area of city from March 2016 to March 2019. Furthermore, there were LL clusters in the northeastern area of the city from March 2016 to March 2019, which had the smallest areas with high priority (Fig. 5).

The first scatter plot shows the CL incidence for 2016-2017 with a Moran's value of 0.236 that should be interpreted as a spatial pattern with a cluster tendency (HH and LL). The second and third scatter plots revealed the $C L$ incidence by Moran's value of 0.296 and 0.231 during 2017-2018 and 2018-2019, respectively. On the lower end of the spectrum, there is a much smaller spread in the CL incidence over the three-year period (Fig. 6).

\section{Discussion}

The main aim of this study was to explore the spatial-time patterns of $C L$ incidence using two spatial analysis techniques: local Moran's clustering analysis on crude incidence rate and spatial empirical Bayesian smoothed rate. To the best of our knowledge, this is the first study in the city of Mashhad to assess spatial patterns of $\mathrm{CL}$ incidence using these two approaches at the finest geographical level (neighborhoods). In the present study, our objective was to understand the spatial and temporal trends of $\mathrm{CL}$. Hence, we evaluated the incidence rates of $\mathrm{CL}$, and designed risk maps for the 2016-2019 period using GIS techniques. The results identified the southwestern areas of the city as high-risk areas. Furthermore, northeastern areas were identified as low-risk CL areas. Also, temporal variation of CL revealed that $\mathrm{CL}$ incidence rates have steadily declined during the study period. The declining trend of $\mathrm{CL}$ is also reported in a previous study, which assessed CL incidence during the last four years in Mashhad (45). An important factor that may have contributed to the steady decline of CL infection rates over last years is the new municipal waste management system. This is further explained by the fact that this new system has facilitated the collection of construction waste in urban areas, which is an attraction site for sand flies breeding. Also, a recent change in climate in Mashhad, which has resulted in heavy rains and floods, may have been another contributing factor in the decline of $\mathrm{CL}$ infections. This is because the heavy precipitation can wash away sand flies' eggs and larva.

Khorasn-Razavi province is an area of long-standing hyper-endemic CL with a reported prevalence rate of $64 / 100,000$ in 2013 (46). Mashhad, the province capital, is one of the main CL endemic areas with a high prevalence rate (45). Despite previous attempts to prevent and control leishmaniasis, new cases of $\mathrm{CL}$ are reported every year, highlighting the need for further interventions (47). The first step in the design of $\mathrm{CL}$ control and prevention programs is the identification of high-risk areas. Taking advantage of GIS techniques and spatial analysis plays an important role in the identification and visualization of highly affected regions. Previous studies assessed the spatial distribution of CL in some parts of Iran $(24,25$, $27,29)$ and also other endemic countries $(22,23)$, in an attempt to identify areas of high priority. In some studies, the clustering method has been used for spatial analysis of $\mathrm{CL}$ incidence in different countries and provinces $(23,24,27,48)$, while other studies have used different approaches such as geographic 
weighted regression (GWR), ordinary least square (OLS) regression, bivariate and multivariate logistic regression to evaluate the relative environmental factors $(29,49)$. The present study revealed a high incidence rate of $C L$ in southwestern Mashhad using both crude incidence rate and spatial empirical Bayes rate analysis.

Previous epidemiological studies in Mashhad revealed the regions with high incidence. The Ab-o-Bargh region had the highest prevalence of $C L$ in 2001. This finding is in line with the results of the present study. Some socio-epidemiological risk factors were identified for high incidence in this region, which include density of population, stray dogs, and some cultural and economic factors (13). Some studies also revealed a high frequency of CL in the cities of Neishabour (50) and Torghabeh-Shandiz (16). Both of these cities are located to the west of Mashhad, and these results reinforce the findings of the present study. A previous study indicated that the high frequency of $\mathrm{CL}$ was due to deforestation or human migration (51). Our findings strengthen this hypothesis since there has been fast and uncontrolled migration to southwestern areas of Mashhad, where we found a high rate of CL incidence. Migration is potentially a contributing factor since it can provide the means for disease transmission. Another important factor, which was also identified in the previous studies and contributes to the increase in $\mathrm{CL}$ incidence, is urbanization (52). Human activities such as construction of dams, roads, and new residential complexes can cause high $\mathrm{CL}$ incidence rates. Also, living in the marginalized areas of the city, which suffer from poor health services, can be regarded as another risk factor for $C L(31,53)$.

Ab-o-Bargh and Torghabeh-Shandiz regions are mountainous areas. As such, they are home to rodent nests and carnivores, which are the main reservoirs of leishmaniasis. Moreover, the two aforementioned neighborhoods are humid, which provide a contributing environment for sand fly breeding (54). These may be regarded as considerable environmental factors that can be taken into account for future implementation of control strategies.

\section{Limitations}

There are some limitations for this study. Iran has a passive system for CL surveillance, which may contribute to underreporting of $\mathrm{CL}$ cases. Also, there may exist cases with small lesions who might not refer to health centers. This can affect the number of diagnosed and reported cases in some areas. Furthermore, the contribution of social and environmental factors was not investigated in this study, which can be the topic of future studies. Despite these limitations, the data used in this study were sufficiently robust for spatial-time analysis of CL cases, and for identifying the priority areas for surveillance in Mashhad.

\section{Conclusions}

The present study provides a vivid depiction of the spatial-time spread of $\mathrm{CL}$, with most cases concentrated in the southwestern region of Mashhad during the three-year study period. In our study, high-risk and low-risk areas were identified using Local Moran's / on crude incidence rate and empirical 
Bayesian smoothed rate. These findings indicate that GIS is a feasible approach for identifying spatialtime patterns of particular infectious diseases. The presented approach can be applied to other geographical areas, or other forms of disease. Our results are designed to help policymakers implement more targeted interventions for disease prevention and control. Furthermore, these findings can be used to hold community training programs in high-risk areas so as to improve the awareness and skills of individuals who are at the higher risk of disease for using personal protective equipment and home spraying of sand flies. Furthermore, identifying high risk areas can help in effective health source allocations such as distribution of free window grids.

\section{Abbreviations}

CL: Cutaneous leishmaniasis; GIS: Geographic Information Systems; HH: High-High; LL: Low-Low; HL: High-Low; LH: Low-High;

\section{Declarations}

\section{Ethics approval and consent to participate}

This study has been approved by the Ethical Committee of Mashhad University of Medical Sciences (number=971558).

\section{Consent for publication}

Not applicable.

\section{Availability of data and materials}

All data presented in the study are available from the corresponding authors.

\section{Competing interests}

The authors declare that they have no competing interests.

\section{Funding}

This study received funding from Mashhad University of Medical Sciences (number= 971558). The funder provided the cost of geocoding and cleaning the data.

\section{Authors' contributions}


E.M and M.F provided the data. A.R.R geocoded the data. R.S prepared and cleaned the data. B.K, N.F and A.M conducted spatial analyses. B.K and N.F drafted the manuscript. M.M, E.M and D.H revised the text. B.K designed the study and was the research leader. The authors read and approved the final manuscript.

\section{Acknowledgements}

We would like to thank Mashhad University of Medical Sciences for funding this study.

\section{References}

1. Alvar J, Vélez ID, Bern C, Herrero M, Desjeux P, Cano J, et al. Leishmaniasis worldwide and global estimates of its incidence. PloS one. 2012;7(5):e35671.

2. World Health Organization: Leishmaniasis [Available from: https://www.who.int/news-room/factsheets/detail/leishmaniasis.

3. Desjeux P. Leishmaniasis: public health aspects and control. Clinics in dermatology. 1996;14(5):41723.

4. Status of endemicity of cutaneous leishmaniasis, worldwide, 2018 [Available from: https://www.who.int/leishmaniasis/burden/en/.

5. Galgamuwa LS, Dharmaratne SD, Iddawela D. Leishmaniasis in Sri Lanka: spatial distribution and seasonal variations from 2009 to 2016. Parasites \& vectors. 2018;11(1):60.

6. Ferro C, Marín D, Góngora R, Carrasquilla MC, Trujillo JE, Rueda NK, et al. Phlebotomine vector ecology in the domestic transmission of American cutaneous leishmaniasis in Chaparral, Colombia. The American journal of tropical medicine and hygiene. 2011;85(5):847-56.

7. Bari AU, Rahman SB. Many faces of cutaneous leishmaniasis. Indian Journal of Dermatology, Venereology, and Leprology. 2008;74(1):23.

8. Bilgic-Temel A, Murrell DF, Uzun S. Cutaneous leishmaniasis: a neglected disfiguring disease for women. International Journal of Women's Dermatology. 2019;5(3):158-65.

9. Piroozi B, Moradi G, Alinia C, Mohamadi P, Gouya MM, Nabavi M, et al. Incidence, burden, and trend of cutaneous leishmaniasis over four decades in Iran. Iranian Journal of Public Health. 2019;48(Supple 1):28-35.

10. Behravan M, Moin-Vaziri V, Haghighi A, Rahbarian N, Taghipour N, Abadi A, et al. Molecular identification of Leishmania species in a re-emerged focus of cutaneous leishmaniasis in Varamin district, Iran. Journal of Arthropod-Borne Diseases. 2017;11(1):124.

11. Salahi-Moghaddam A, Khoshdel A, Hanafi-Bojd A-A, Sedaghat M-M. Mapping and review of leishmaniasis, its vectors and main reservoirs in Iran. Journal of Kerman University of Medical Sciences. 2015;22(1):83-104.

12. Norouzinezhad F, Ghaffari F, Norouzinejad A, Kaveh F, Gouya MM. Cutaneous leishmaniasis in Iran: results from an epidemiological study in urban and rural provinces. Asian Pacific journal of tropical 
biomedicine. 2016;6(7):614-9.

13. Shamsian SAA, Mohajery M, Fata A, Sadabadi F, Berenji F, Mastroeni $P$, et al. Changes in the Epidemiology of Cutaneous Leishmaniasis in Northeastern Iran. Türkiye Parazitolojii Dergisi. 2020;44(1):52.

14. Farash BRH, Mohajery M, Fata A, Shamsian SA, Rezaee A, Yazdanpanah MJ. Anthroponotic cutaneous leishmaniasis in torghabeh-shandiz, a region with rural texture (a molecular study). Jundishapur Journal of Microbiology. 2013;6(10):1R.

15. Valizadeh M, DALIMI AH, Fata A, Jafari M, Khamesipour A, Mohebali M. A study on leishmania species causing cutaneous leishmaniasis inmashhad using specific monoclonal antibodies. 2005.

16. Khajedaluee M, Yazdanpanah MJ, SeyedNozadi S, Fata A, Juya MR, Masoudi MH, et al. Epidemiology of cutaneous leishmaniasis in population covered by Mashhad University of Medical Sciences in 2011. Medical Journal of Mashhad university of Medical sciences. 2014;57(4):647-54.

17. Masimalai P. Remote sensing and Geographic Information Systems (GIS) as the applied public health and environmental epidemiology. Int J Med Sci Public Health. 2014;3(12):1430.

18. Palaniyandi M. GIS for disease surveillance and health information management in India. Geospatial Today. 2014;13(5):44-6.

19. Barbosa DS, Belo VS, Rangel MES, Werneck GL. Spatial analysis for identification of priority areas for surveillance and control in a visceral leishmaniasis endemic area in Brazil. Acta Tropica. 2014; 131:56-62.

20. Melchior LAK, Brilhante AF, Chiaravalloti-Neto F. Spatial and temporal distribution of American cutaneous leishmaniasis in Acre state, Brazil. Infectious diseases of poverty. 2017;6(1):99.

21. Gálvez R, Descalzo M, Miró G, Jiménez M, Martín O, Dos Santos-Brandao F, et al. Seasonal trends and spatial relations between environmental/meteorological factors and leishmaniosis sand fly vector abundances in Central Spain. Acta tropica. 2010;115(1-2):95-102.

22. Maia-Elkhoury ANS, E. Yadón Z, Idali Saboyá Díaz M, de Fátima de Araújo Lucena F, Gerardo Castellanos L, J. Sanchez-Vazquez M. Exploring spatial and temporal distribution of cutaneous leishmaniasis in the Americas, 2001-2011. PLoS neglected tropical diseases. 2016;10(11):e0005086.

23. Cardoso DT, de Souza DC, de Castro VN, Geiger SM, Barbosa DS. Identification of priority areas for surveillance of cutaneous leishmaniasis using spatial analysis approaches in Southeastern Brazil. BMC infectious diseases. 2019;19(1):318.

24. Abedi-Astaneh F, Hajjaran H, Yaghoobi-Ershadi MR, Hanafi-Bojd AA, Mohebali M, Shirzadi MR, et al. Risk mapping and situational analysis of cutaneous leishmaniasis in an endemic area of Central Iran: a GIS-based survey. PLoS One. 2016;11(8):e0161317.

25. Dehghani A, Lotfi MH, Falahzadeh H, Vahdat K, Shabani Z. Epidemiological Study and Spatial Modeling of Cutaneous Leishmaniasis in Bushehr Province using the Geographic Information System (GIS) during 2011-2015. Journal of Community Health Research. 2019;8(3):156-63. 
26. Khademvatan S, Salmanzadeh S, Foroutan-Rad M, Bigdeli S, Hedayati-Rad F, Saki J, et al. Spatial distribution and epidemiological features of cutaneous leishmaniasis in southwest of Iran. Alexandria Journal of Medicine. 2017;53(1):93-8.

27. Ramezankhani R, Sajjadi N, Esmaeilzadeh RN, Jozi SA, Shirzadi MR. Spatial analysis of cutaneous leishmaniasis in an endemic area of Iran based on environmental factors. Geospatial health. 2017.

28. Shirzadi MR, Javanbakht M, Jesri N, Saghafipour A. Spatial Distribution of Cutaneous Leishmaniasis Cases Referred to Health Centers of Three Khorasan Provinces in Iran Using Geographical Information System. Iranian Journal of Public Health. 2019;48(10):1885.

29. Ali-Akbarpour M, Mohammadbeigi A, Tabatabaee SHR, Hatam G. Spatial analysis of ecoenvironmental risk factors of cutaneous leishmaniasis in southern Iran. Journal of cutaneous and aesthetic surgery. 2012;5(1):30.

30. Shirzadi MR, Javanbakht M, Vatandoost H, Jesri N, Saghafipour A, Fouladi-Fard R, et al. Impact of Environmental and Climate Factors on Spatial Distribution of Cutaneous Leishmaniasis in Northeastern Iran: Utilizing Remote Sensing. Journal of Arthropod-Borne Diseases. 2020;14(1):56.

31. Galavizade S, Fata A, Vakili V, Zarean M. Survey the cutaneous leishmaniasis prevalence in Mashhad during the past twenty years (1995-2014) and the effect of environmental risk factors on that. medical journal of mashhad university of medical sciences. 2015;58(9):516-22.

32. Kim D, Sarker M, Vyas P. Role of spatial tools in public health policymaking of Bangladesh: opportunities and challenges. Journal of Health, Population and Nutrition. 2016;35(1):1-5.

33. Parish LC. Andrews' Diseases of the Skin: Clinical Dermatology. JAMA. 2011;306(2):213-.

34. McMaster R. In Memoriam: George F. Jenks (1916-1996). Cartography and Geographic Information Systems. 1997;24(1):56-9.

35. Efron B. Large-scale inference: empirical Bayes methods for estimation, testing, and prediction: Cambridge University Press; 2012.

36. Bailey TC, Gatrell AC. Interactive spatial data analysis: Longman Scientific \& Technical Essex; 1995.

37. Kumar VS, Devika S, George S, Jeyaseelan L. Spatial mapping of acute diarrheal disease using GIS and estimation of relative risk using empirical Bayes approach. Clinical epidemiology and global health. 2017;5(2):87-96.

38. Marshall RJ. Mapping disease and mortality rates using empirical Bayes estimators. Journal of the Royal Statistical Society: Series C (Applied Statistics). 1991;40(2):283-94.

39. Manton KG, Woodbury MA, Stallard E, Riggan WB, Creason JP, Pellom AC. Empirical Bayes procedures for stabilizing maps of US cancer mortality rates. Journal of the American Statistical Association. 1989;84(407):637-50.

40. Anselin L. Local indicators of spatial association-LISA. Geographical analysis. 1995;27(2):93-115.

41. Halimi L, Bagheri N, Hoseini B, Hashtarkhani S, Goshayeshi L, Kiani B. Spatial analysis of colorectal cancer incidence in Hamadan Province, Iran: a retrospective cross-sectional study. Applied Spatial Analysis and Policy. 2019:1-11. 
42. Goshayeshi L, Pourahmadi A, Ghayour-Mobarhan M, Hashtarkhani S, Karimian S, Dastjerdi RS, et al. Colorectal cancer risk factors in north-eastern Iran: A retrospective cross-sectional study based on geographical information systems, spatial autocorrelation and regression analysis. Geospatial health. 2019;14(2).

43. Bagheri N, Furuya-Kanamori L, Doi SA, Clements AC, Sedrakyan A. Geographical outcome disparities in infection occurrence after colorectal surgery: An analysis of 58,096 colorectal surgical procedures. International Journal of Surgery. 2017;44:117-21.

44. Anselin L. The Moran scatterplot as an ESDA tool to assess local instability in spatial. Spatial Analytical. 1996;4:111.

45. Abdolmajid Fata Bk, Amene Raouf Rahmati, Maryam Tayefi, Faezaeh Moradinejad, Elham moghaddas. Epidemiological Status of Cutaneous Leishmaniasis during Past 4 Years (2015-18) in Mashhad, Northeastern Iran. Zahedan Journal of Research in Medical Sciences In press. 2020.

46. Akhoundi M, Mohebali M, Asadi M, Mahmodi MR, Amraei K, Mirzaei A. Molecular characterization of Leishmania spp. in reservoir hosts in endemic foci of zoonotic cutaneous leishmaniasis in Iran. Folia Parasitologica. 2013;60(3):218.

47. Yaghoobi-Ershadi M. Phlebotomine sand flies (Diptera: Psychodidae) in Iran and their role on Leishmania transmission. Journal of arthropod-borne diseases. 2012;6(1):1.

48. Machado-Coelho G, Assunção R, Mayrink W, Caiaffa WT. American cutaneous leishmaniasis in Southeast Brazil: space-time clustering. International journal of epidemiology. 1999;28(5):982-9.

49. Seid A, Gadisa E, Tsegaw T, Abera A, Teshome A, Mulugeta A, et al. Risk map for cutaneous leishmaniasis in Ethiopia based on environmental factors as revealed by geographical information systems and statistics. Geospatial health. 2014;8(2):377-87.

50. Sakhaei S, Darrudi R, Motaarefi H, Sadagheyani H. Epidemiological Study of Cutaneous Leishmaniasis in Neyshabur County, East of Iran (2011-2017). Open Access Maced J Med Sci. 2019 Nov 15; 7 (21): 3710-3715. 2019.

51. Soares VB, Almeida ASd, Sabroza PC, Vargas WP. Epidemiological surveillance of tegumentary leishmaniasis: local territorial analysis. Revista de saude publica. 2017;51:51.

52. Nascimento BWL, Saraiva L, Neto RGT, e Meira PCLS, de Castilho Sanguinette C, Tonelli GB, et al. Study of sand flies (Diptera: Psychodidade) in visceral and cutaneous leishmaniasis areas in central western of Minas Gerais state-Brazil. Acta Tropica. 2013;125(3):262-8.

53. Quintana MG, Santini MS, Cavia R, Martínez MF, Liotta DJ, Fernández MS, et al. Multiscale environmental determinants of Leishmania vectors in the urban-rural context. Parasites \& vectors. 2020;13(1):1-15.

54. Wijerathna T, Gunathilaka N. Diurnal adult resting sites and breeding habitats of phlebotomine sand flies in cutaneous leishmaniasis endemic areas of Kurunegala District, Sri Lanka. Parasites \& Vectors. 2020;13(1):1-10.

\section{Figures}




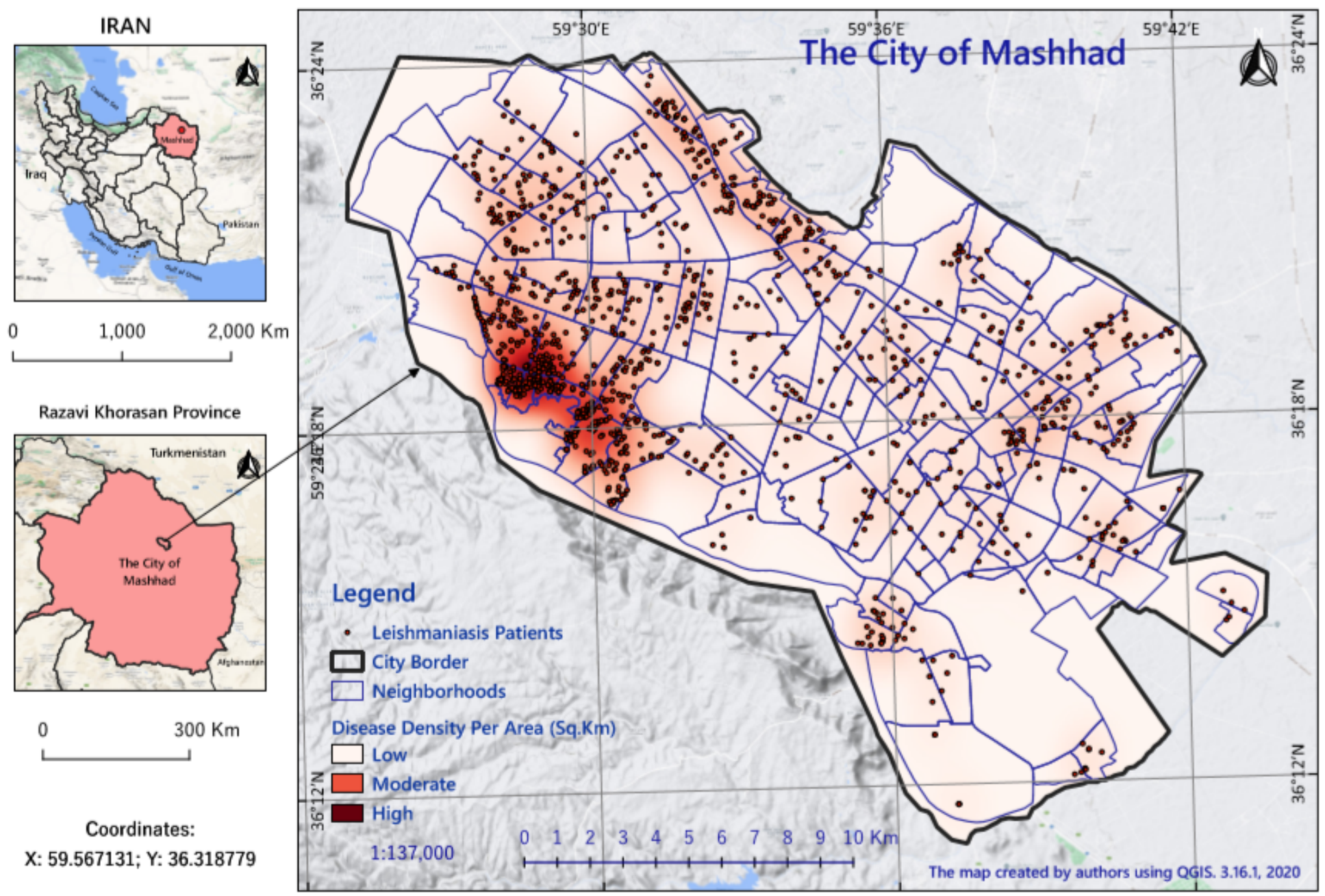

Figure 1

Map of neighborhoods and distribution of cutaneous leishmaniasis patients in the city of Mashhad during 2016-2019 


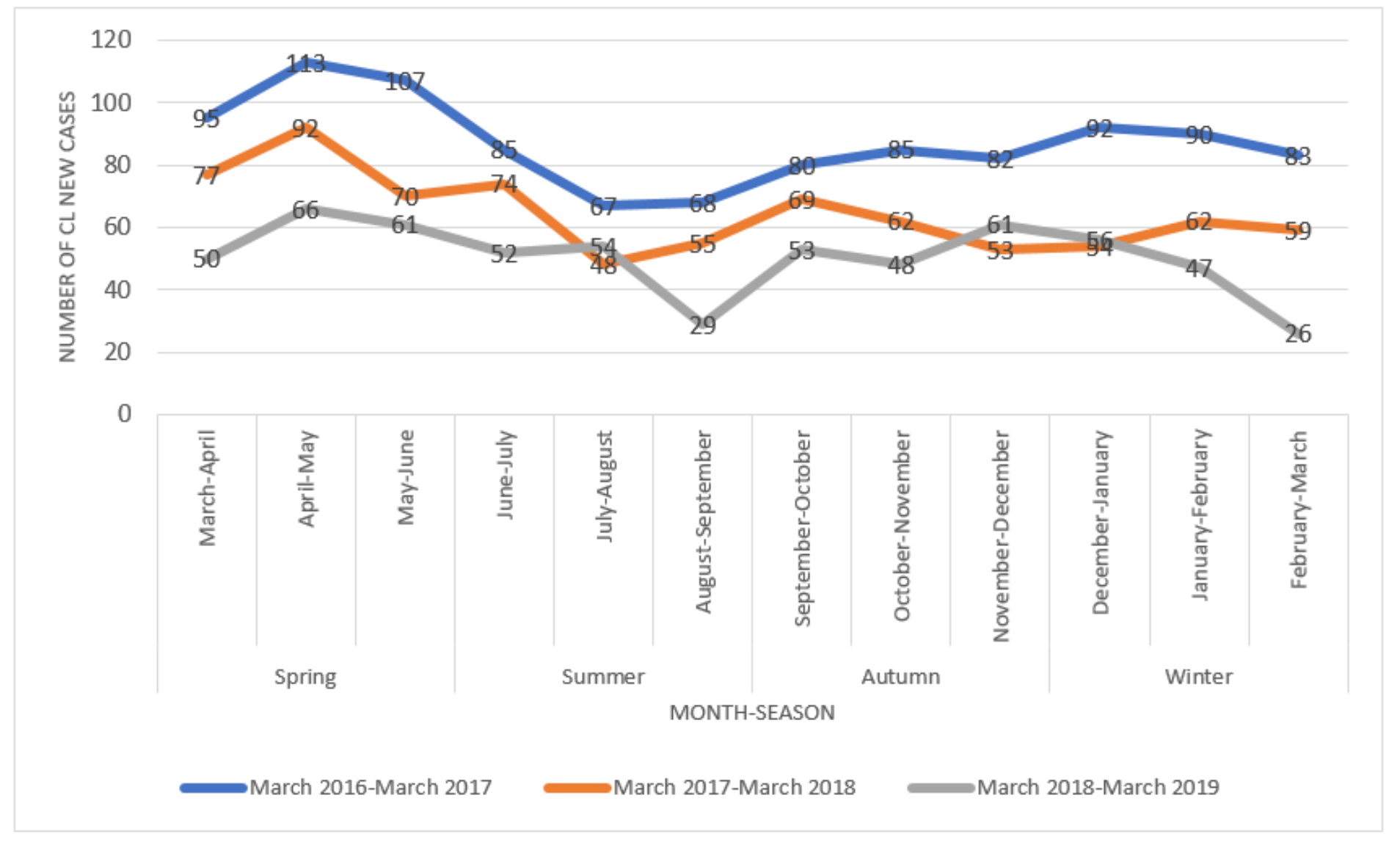

\section{Figure 2}

Temporal distribution of cutaneous leishmaniasis (CL) in the city of Mashhad from 2016 to 2019
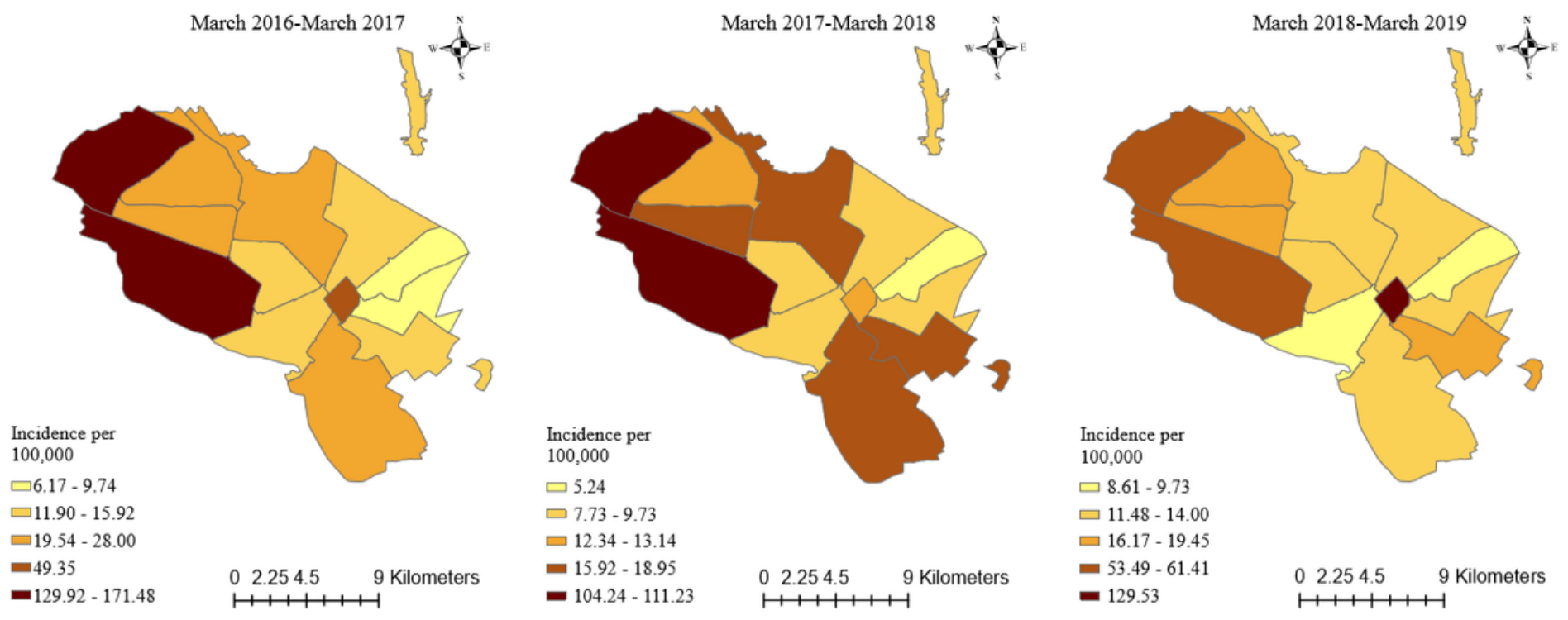

\section{Figure 3}

Cutaneous leishmaniasis incidence map at the district level in the city of Mashhad during 2016-2019 

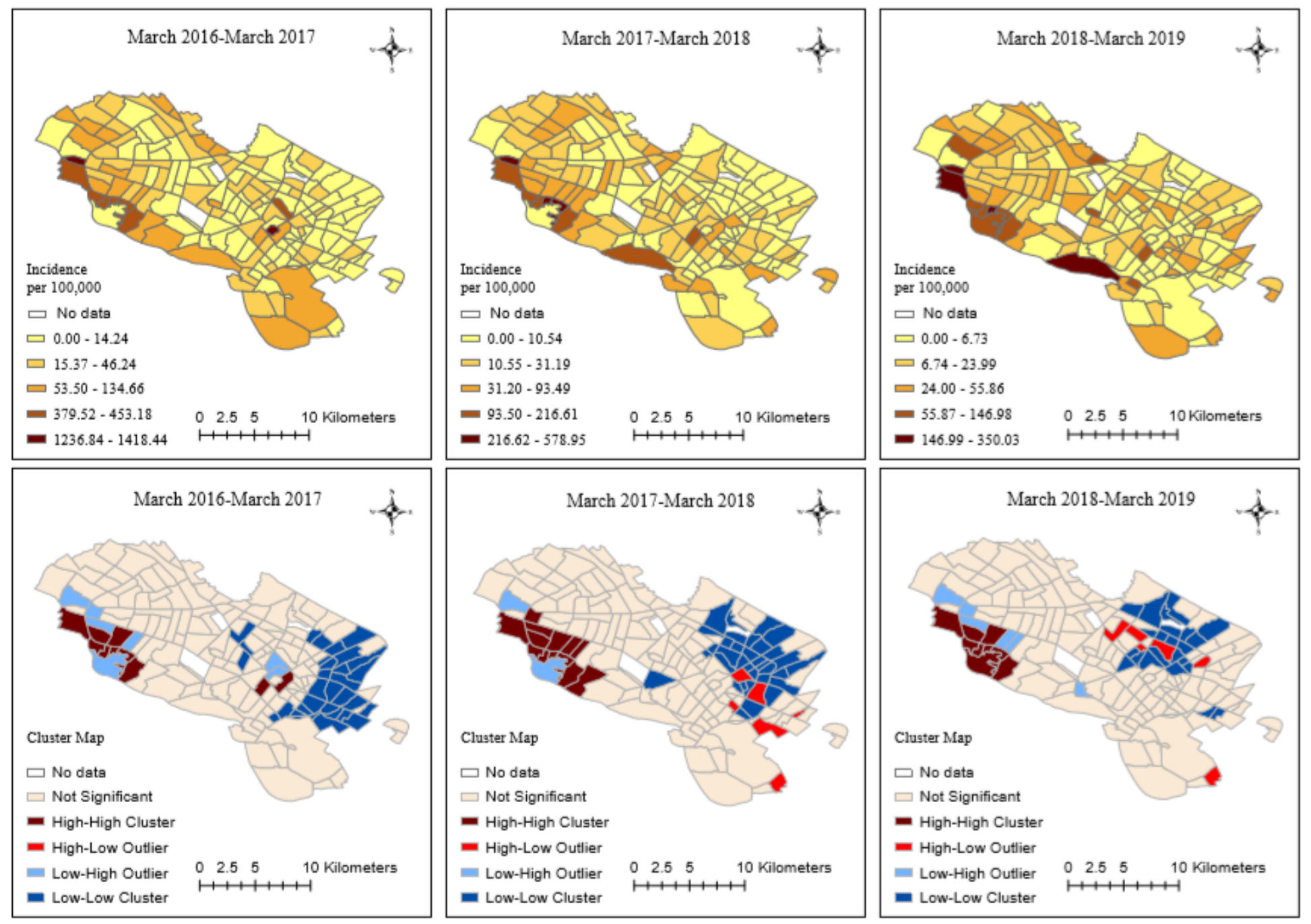

\section{Figure 4}

Crude incidence rates of cutaneous leishmaniasis per 100,000 at the neighborhood level in the city of Mashhad, during 2016-2019 - Clustering Maps by crude incidence rate 

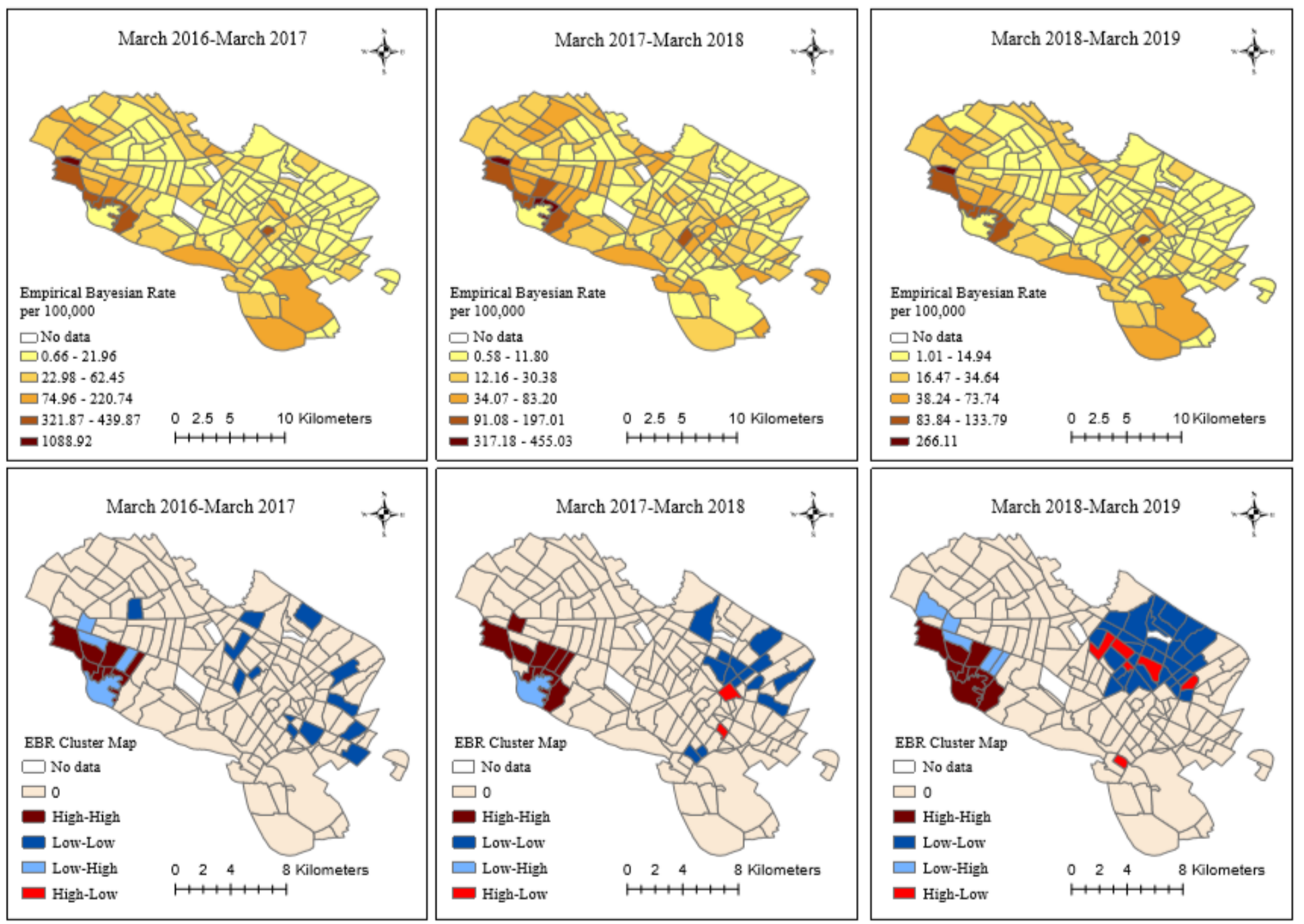

Figure 5

Smoothed incidence rates of cutaneous leishmaniasis per 100,000 at the neighborhood level in the city of Mashhad, during 2016-2019 - Clustering Maps by smoothed Bayesian incidence rate

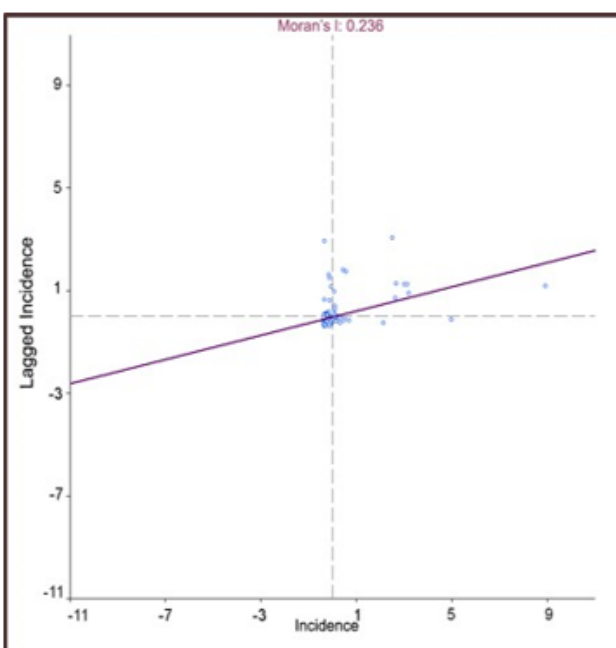

Cutaneous leishmaniasis Incidence (2016-2017)

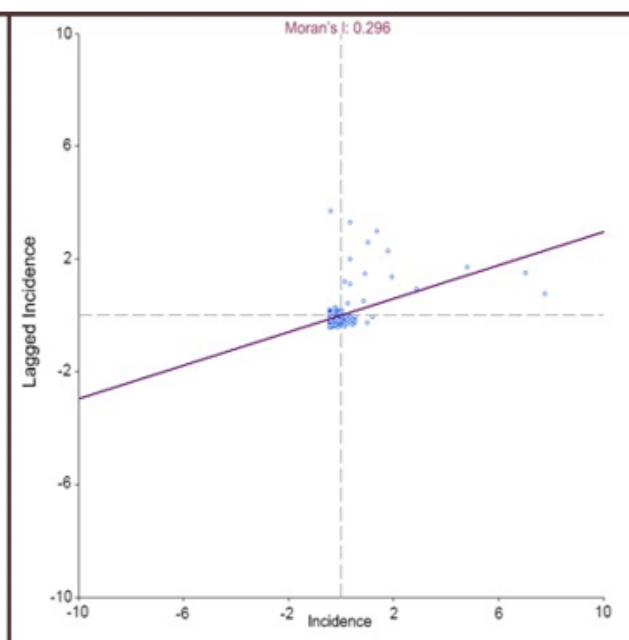

Cutaneous leishmaniasis Incidence (2017-2018)

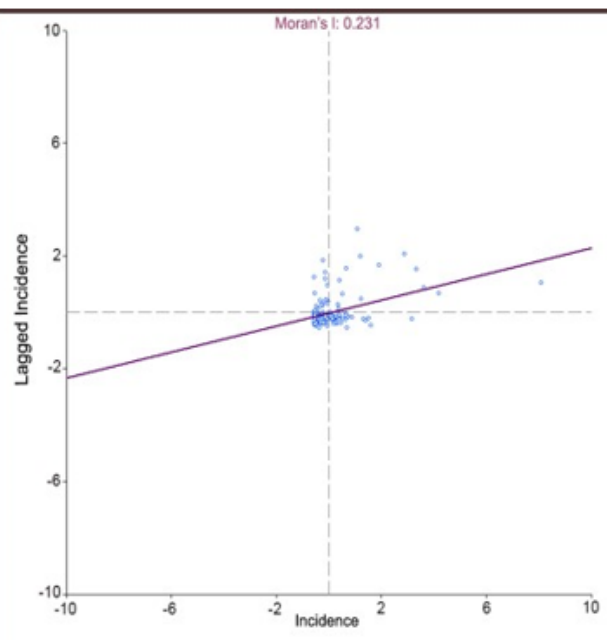

Cutaneous leishmaniasis Incidence (2018-2019) 
Figure 6

Moran's scatter plots for CL cluster maps 